\title{
Mesenchymal stem cells-seeded bio-ceramic construct for bone regeneration in large critical-size bone defect in rabbit
}

\author{
Maiti SK ${ }^{1}$, Ninu $\mathrm{AR}^{2}$, Sangeetha $\mathrm{P}^{2}$, Mathew $\mathrm{DD}^{2}$, Tamilmahan $\mathrm{P}^{2}$, Kritaniya $\mathrm{D}^{3}$, Kumar $\mathrm{N}^{1}$, Hescheler $\mathrm{J}^{4}$
}

Bone marrow derived mesenchymal stem cells (BMSC) represent an attractive cell population for tissue engineering purpose. The objective of this study was to determine whether the addition of recombinant human bone morphogenetic protein (rhBMP-2) and insulin-like growth factor (IGF-1) to a silica-coated calcium hydroxyapatite (HASi) - rabbit bone marrow derived mesenchymal stem cell (rBMSC) construct promoted bone healing in a large segmental bone defect beyond standard critical -size radial defects $(15 \mathrm{~mm})$ in rabbits. An extensively large $30 \mathrm{~mm}$ long radial ostectomy was performed unilaterally in thirty rabbits divided equally in five groups. Defects were filled with a HASi scaffold only (group B); HASi scaffold seeded with rBMSC (group C); HASi scaffold seeded with rBMSC along with rhBMP-2 and IGF-1 in groups D and

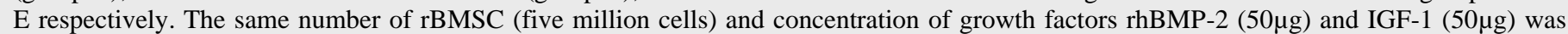
again injected at the site of bone defect after 15 days of surgery in their respective groups. An empty defect served as the control group (group A). Radiographically, bone healing was evaluated at 7, 15, 30, 45, 60 and 90 days post implantation. Histological qualitative analysis with microCT $(\mu-\mathrm{CT})$, haematoxylin and eosin $(\mathrm{H} \& \mathrm{E})$ and Masson's trichrome staining were performed 90 days after implantation. All rhBMP-2-added constructs induced the formation of well-differentiated mineralized woven bone surrounding the HASi scaffolds and bridging bone/implant interfaces as early as eight weeks after surgery. Bone regeneration appeared to develop earlier with the rhBMP-2 constructs than with the IGF-1 added construct. Constructs without any rhBMP-2 or IGF-1 showed osteoconductive properties limited to the bone junctions without bone ingrowths within the implantation site. In conclusion, the addition of rhBMP-2 to a HASi scaffold could promote bone generation in a large critical-size-defect.

Key Words: Mesenchymal stem cells, Recombinant human bone morphogenetic protein, Insulin like growth factor, Silica-coated calcium

\section{Introduction}

Despite the benefits that minimally invasive surgery and osteosynthesis have brought to fracture management and bone healing, there are still many circumstances where bone healing remains challenging ${ }^{[1]}$. Large bone defects are serious complications that are most commonly caused by extensive trauma, tumour, infection, or congenital musculoskeletal disorders. In nonunion cases repairing of bone defects with composite biomaterials as defect filler can promote bone regeneration ${ }^{[2]}$. Currently, the gold standard for bone regeneration is the use of autogenous bone graft. In order to avoid morbidity at the donor site or if large amount of autogenous bone is needed, bone substitution materials can be used $^{[3]}$.

Bone substitution materials can be combined with cells such as mesenchymal stem cells (MSCs) to increase bone formation ${ }^{[4]}$. Bone-marrow-derived mesenchymal stem cells (BMSCs) represent an attractive cell population for tissue regeneration ${ }^{[5]}$. Bone marrow stem cells are pluripotent cells that have been used to facilitate bone repair because of their capability of differentiating into osteoblasts ${ }^{[6]}$. Several studies on the regeneration of bone have shown that cultured BMSCs, seeded on different bioabsorbable implants are able to induce bone formation in vivo and lead to improved healing of critical-size $\operatorname{defects}^{[4,5]}$.
Calcium phosphate bone substitutes such as hydroxyapatite (HA) and tricalcium phosphate (TCP) are currently used for bone substitution in many different clinical applications such as repair of bone defects after trauma or tumour and bone augmentation in spinal arthrodesis ${ }^{[7,8]}$. Although, these bone substitutes are osteoconductive, they often lack the osteogenecity needed to support bone healing in large defects and are slowly degraded in the body ${ }^{[1]}$. Studies had shown that coating of hydroxyapatite with a calcium silicate layer could encourage cell proliferation and osteogenic differentiation of human bone marrow-derived stromal cells $^{[9]}$. Silica-calcium phosphate composite in comparison to calcium phosphate-rich biomaterials has a faster resorption rate owing to greater dissolution of $\mathrm{Si}$ ions ${ }^{[10]}$.

The use of several growth factors have been studied in bone repair and these factors are known to play a role in differentiation of mesenchymal progenitor cells specific lineages like endothelial cells or osteoblasts ${ }^{[11,12]}$. Bone morphogenetic protein-2 (BMP-2) has been shown to accelerate bone healing in humans and animal models ${ }^{[13,14]}$. BMP-2 acts by osteoinduction and is involved in the differentiation of mesenchymal progenitor cells into osteoblasts ${ }^{[1,14]}$. The addition of recombinant human BMP (rhBMP-2) to a self-cross linkable cellulose hydrogels/biphasic calcium phosphate granules construct promotes bone regeneration in a critical-size segmental defect model of nonunion in $\operatorname{dogs}^{[1]}$. It is also reported that protein, insulin-like growth factor (IGF-1) stimulates direct migration of human mesenchymal 
progenitor cells (MPC) and contributes to the recruitment of MPC in bone formation and bone healing ${ }^{[15]}$.

Transplantation of human mesenchymal stem cells in a nonautogenous setting for bone regeneration in a rabbit critical-size defect $(15 \mathrm{~mm})$ has been reported ${ }^{[5]}$, but the regeneration potential of autogenous MSC with or without growth factors beyond standard critical-size defect was not reported. So, in the current study, we investigate the potential of cultured autogenic rabbit bone marrowderived mesenchymal stem cells (rBMSC) seeded in a triphasic composite bio-scaffold (namely $\mathrm{HASi}$ ) to generate new bone formation in an extensively large $(30 \mathrm{~mm})$ segmental diaphyseal bone defect in rabbit model with or without growth factors rhBMP-2 and IGF-1.

\section{Materials and Methods}

\section{Experimental design}

The study was conducted on 30 female adult New Zealand White rabbits $(2.86 \pm 0.38 \mathrm{~kg}, 6-9$ months old) divided into five equal groups (each $n=6$ ). They were kept in individual cages, fed a standard diet and allowed free mobilization during the study. The groups were compared as follows: The radial $30-\mathrm{mm}$ segmental diaphyseal defect was filled with a HASi scaffold only (group B); HASi scaffold seeded with autogenous rBMSC (group C); HASi scaffold-seeded with autogenous rBMSC along with rhBMP-2 (group D) and HASi scaffold-seeded with autogenous rBMSC along with IGF-1 (group E). An empty defect served as the control group (group A).

The same number of rBMSC (five millions cells) and concentration of growth factors rhBMP-2 $(50 \mu \mathrm{g})$ and IGF-1 (50 $\mu \mathrm{g})$ were again injected around the bone defect on day 15 postsurgery in their respective groups.

Animals were treated in compliance with the guiding principles in the "Care and Use of Animals" policy of the authors' institutions. The institute Animal Ethical Committee for animal experiments has approved the design of the experiment.

Preoperatively, hematology was performed to ensure the absence of systemic diseases. The rabbits had no signs of bone or joint disease on the relevant limbs, as assessed by clinical examination and preoperative radiographs.

\section{Bioceramic scaffold}

A triphasic composite bio-scaffold (calcium silicate, hydroxyapatite and tricalcium phosphate) namely HASi with elements in the following percentage: calcium-66.36\%, Phosphorus- $25.35 \%$, Silicon$8.29 \%$ and porosity of $50-500 \mu \mathrm{m}$ was used as ceramic bloc. Each HASi block was of $30 \mathrm{~mm}$ long and $5 \mathrm{~mm}$ in diameter were produced in an emulsion process as described earlier ${ }^{[16]}$.

\section{Isolation and culture of $r B M S C$}

Isolation and culture of rBMSC was performed as described earlier ${ }^{[17]}$. The rBMSCs at passage- 3 were used for this experiment.

\section{Seeding of rBMSC into HASi ceramic blocks}

The HASi bioscaffold were incubated at $4^{0} \mathrm{C}$ in $50 \mu \mathrm{M} \mathrm{ml}{ }^{-1}$ fibronectin solution diluted in PBS for $24 \mathrm{~h}$. Five million passage-3 rBMSC were resuspended in $4 \mathrm{ml}$ of culture medium (DMEM) and transferred into a $5 \mathrm{ml}$ tube. The HASi blocks were placed into the medium containing the cells. After $1.5 \mathrm{~h}$ of continuous spinning at $37^{0} \mathrm{C}(35 \mathrm{rpm})$, the ceramic blocks were placed into a 6-well plate.
The medium containing the cells was centrifuged twice at $800 \mathrm{~g}$ for 5 min, and the resulting cell pellet was re-suspended in $70 \mu 1$ of culture medium and applied to the ceramic blocks. This MSC-seeded ceramic scaffold was used in group C. Tissue engineered bone construct for group D consisted of seeded HASi scaffold along with $50 \mu \mathrm{g}$ of rhBMP-2 suspended in $250 \mu \mathrm{l}$ of DMEM, injected at the implant site, whereas, in group E, seeded HASi scaffold along with $50 \mu \mathrm{g}$ of IGF-1 suspended in $250 \mu \mathrm{l}$ of DMEM injected at the implant site. In group B, only HASi block/scaffold was used at the implant site.

\section{Surgical procedure}

All the animals were acclimatized for two weeks to approaching and handling prior to surgery. Under general anesthesia with xylazine hydrochloride $(5 \mathrm{mg} / \mathrm{kg}, \mathrm{IM})$ and ketamine hydrochloride $(50 \mathrm{mg} / \mathrm{kg}$, IM) and strict surgical asepsis, a unilaterally critical-size bone defects were created in the radial diaphysis. A $4 \mathrm{~cm}$ super medial incision over the radius was given, soft tissue was dissected and the bone exposed by gentle retraction of the muscles. A Hohmann retractor was placed between ulna and radius to protect the ulna. A $30 \mathrm{~mm}$ segmental osteo-periosteal diaphyseal defect was created with an oscillating bone saw that was continuously cooled by irrigation with $0.9 \%$ sterile cold saline solution. The periosteum was removed with the bone and $5 \mathrm{~mm}$ of periosteum was stripped from each side of the remaining radius. The bone gap was irrigated with sterile physiological saline solution and ceramic block (or nothing) fitted into the gap as per treatment protocol for different groups. Muscles, fascia and skin were separately closed over the defect with 4-0 resorpable sutures. A wooden splint with bandage was applied on the test bone for one week.

\section{Postoperative care}

Postoperatively, ceftriaxone sodium $(22 \mathrm{mg} / \mathrm{kg}$, IM) administered intramuscularly for five days. Analgesic meloxicam $(0.2 \mathrm{mg} / \mathrm{kg}$, IM) was given for the first 3 postoperative days. There were no differences in quantity of meloxicam administered between the groups. Water and food were supplied ad libitum. After 90 days of experiment, the animals were sacrificed.

\section{Radiological assessment}

Craniocaudal and mediolateral radiographic view of each ostectomy site was assessed immediately after surgery to monitor the position of the implants, and at 7, 15, 30, 45, 60 and 90 days postoperatively to assess bone formation and bone union with the host bone at both proximal and distal junctions. The radiographs were graded with slight modification of the scoring system reported by Yang et $a l^{[18]}$ (Table 1). For each category mean scores were calculated. These mean scores of each time interval were added separately for obtaining the total radiographic scores. Overall radiographic scores for each group were calculated by adding mean scores for each group at different intervals. Resorption of the implant was assessed qualitatively.

\section{Microcomputed tomography assessment}

Microcomputed tomography $(\mu \mathrm{CT})$ technique was used for evaluation of three dimensional (3-D) trabecular new bone formation in different treatment groups. At day 90 after implantation, the constructs were explanted after euthanizing the animals, preserving both proximal and distal interfaces. Images were acquired under $70 \mathrm{keV}$ voltage with a constant $114 \mu \mathrm{A}$ current. Spatial resolution was limited to 100-200 $\mu \mathrm{m}$. A high- resolution protocol (slice thickness $120 \mu \mathrm{m}$, feed $60 \mu \mathrm{m}$, and pixel size $60 \mu \mathrm{m}$ ) was applied. An X-ray tomographic microscope, Micro-CT 40 was used in this study. 
Table 1. Radiographic grading scale for analyzing the bone healing

\begin{tabular}{|c|c|}
\hline Grading category & Score \\
\hline \multicolumn{2}{|l|}{ Periosteal reaction } \\
\hline None & 0 \\
\hline Minimal (localized to the gaps) & 1 \\
\hline Medium (extends over the gaps or towards ulna) & 2 \\
\hline Moderate ( $1 / 4$ to $1 / 2$ of the defect area) & 3 \\
\hline Full ( $1 / 2$ to full length of defect) & 4 \\
\hline \multicolumn{2}{|l|}{ Osteotomy line } \\
\hline Both the osteotomy lines completely radiolucent & 0 \\
\hline One of the osteotomy lines partially radiolucent & 1 \\
\hline Both the osteotomy lines partially radiolucent & 2 \\
\hline One of the osteotomy lines invisible & 3 \\
\hline Both the osteotomy lines invisible & 4 \\
\hline \multicolumn{2}{|l|}{ Construct appearance } \\
\hline Unchanged/intact & 0 \\
\hline Mild resorption (one localized area) & 1 \\
\hline Moderate resorption (more than one localized area) & 2 \\
\hline Mostly replaced & 3 \\
\hline Fully replaced & 4 \\
\hline
\end{tabular}

\section{Histopathological assessment}

At day 90 post-implantation, the animals were sacrificed. The test bone sections throughout the length of the defect were collected from the site of surgical implant and washed thoroughly with sterile normal saline solution. Fixation was done in $10 \%$ formalin. The bone sections (longitudinal as well as transverse) were subjected to decalcification in Goodling and Stewart solution $(15 \mathrm{ml}$ formic acid, $5 \mathrm{ml}$ formalin and $80 \mathrm{ml}$ distilled water). The decalcified bone sections were stained by $\mathrm{H}$ $\&$ E and Masson's trichrome stain. The sections were examined using a light microscope under different magnification. Bone healing was assessed in each group according to modified Lane and Sandhu ${ }^{[19]}$ and Heiple et $a l^{[20]}$. Histopathological scoring system was presented in Table 2.

Both the radiographic and histological assessments were blindly performed.

\section{Statistical analysis}

All values were expressed as the mean plus or minus standard deviation $( \pm \mathrm{SD})$. All the data were analyzed by analysis of variance (ANOVA) as per standard statistical methods, using SPSS software package (version 16). The various scores were compared with Kruskal-Wallis test. Significance was set for a p-value $<0.05$.

\section{Results}

\section{Clinical assessment}

The food and water intake was returned normal on day 3 in all experimental groups (B, C, D, E) whereas, it was on 6-8 days in control group. Weight bearing on the operated limb was mild to moderate from day 3 after surgery in all groups except group A. Complete weight bearing was seen after removal of wooden splint in all animals of groups B, C, D and E on day 7 after surgery. In the control group the animal did not bear weight normally throughout the experiment period. In three animals of group A, (empty control), a fracture of the treated leg was observed, so these three animals were sacrificed prior to the study endpoint. No animal of this group A was alive at day 90. The implanted biomaterials did not cause any apparent signs of irritation or infection and cutaneous wound healing was uneventful.

\section{Radiographic assessment}

The radiographic scoring was done based on mediolateral view as there was overlapping of the radius by ulna in craniocaudal view. Radiographic scores (mean \pm SD) of construct appearance, osteotomy line, periosteal reaction, total and overall radiographic scores in all five groups at various time intervals are presented in Tables 3. Mediolateral views of radiograph of animals of five groups are presented in Figure 1. Overall radiographic scores of five groups are presented in Figure 2. 
Table 2: Histopathological scoring system

\begin{tabular}{|c|c|c|}
\hline Sl. No & Feature & Score \\
\hline \multicolumn{3}{|c|}{ 1. Osteogenesis } \\
\hline \multicolumn{2}{|r|}{ No osteogenesis } & 0 \\
\hline \multicolumn{2}{|r|}{ Weak osteogenesis } & 1 \\
\hline \multicolumn{2}{|r|}{ Medium osteogenesis } & 2 \\
\hline \multicolumn{2}{|r|}{ Good osteogenesis } & 3 \\
\hline \multicolumn{2}{|r|}{ Perfect osteogenesis } & 4 \\
\hline \multicolumn{3}{|c|}{ 2. Union } \\
\hline \multicolumn{2}{|r|}{ No evidence of union } & 0 \\
\hline & Fibrous union & 1 \\
\hline & Osteochondral union & 2 \\
\hline & Bony union & 3 \\
\hline \multicolumn{2}{|r|}{ Complete organization of graft } & 4 \\
\hline \multirow[t]{6}{*}{3.} & \multicolumn{2}{|l|}{ Marrow } \\
\hline & None in the resected area & 0 \\
\hline & Beginning to appear & 1 \\
\hline & Present in greater than $1 / 2$ defect & 2 \\
\hline & Complete colonization by red marrow & 3 \\
\hline & Mature fatty marrow & 4 \\
\hline \multirow[t]{6}{*}{4.} & \multicolumn{2}{|l|}{ Cancellous bone/medullary bone } \\
\hline & No osseous cellular activity & 0 \\
\hline & Early apposition of new bone & 1 \\
\hline & Active apposition of new bone & 2 \\
\hline & Reorganizing cancellous bone & 3 \\
\hline & Complete re-organization of cancellous bone & 4 \\
\hline \multirow[t]{6}{*}{5.} & \multicolumn{2}{|l|}{ Cortical/compact bone } \\
\hline & None & 0 \\
\hline & Early appearance & 1 \\
\hline & Formation underway & 2 \\
\hline & Mostly re-organized & 3 \\
\hline & Completely formed & 4 \\
\hline
\end{tabular}


Table 3. Radiographic scores (mean $\pm \mathrm{SD}$ ) of construct appearance, osteotomy line, periosteal reaction and total radiographic score at various time interval; overall radiographic scores (Mean $\pm \mathrm{SD}$ ), ${ }^{\mathrm{a}-\mathrm{e}}$ Values in the same column with different superscript letter are significantly different $(\mathrm{P}<0.05)$

\begin{tabular}{|c|c|c|c|c|}
\hline $\begin{array}{l}\text { Group and day } \\
\text { (Interaction) }\end{array}$ & $\begin{array}{c}\text { Construct } \\
\text { appearance }\end{array}$ & Osteotomy line & $\begin{array}{c}\text { Periosteal } \\
\text { reaction }\end{array}$ & Total score \\
\hline$A \times 7$ & No construct & $0.00 \pm 0.00$ & $0.00 \pm 0.00$ & $0.00 \pm 0.00^{\mathrm{a}}$ \\
\hline $\mathrm{B} \times 7$ & $0.00 \pm 0.00$ & $0.00 \pm 0.00$ & $0.00 \pm 0.00$ & $0.00 \pm 0.00^{\mathrm{a}}$ \\
\hline $\mathrm{C} \times 7$ & $0.00 \pm 0.00$ & $0.00 \pm 0.00$ & $0.00 \pm 0.00$ & $0.00 \pm 0.00^{\mathrm{a}}$ \\
\hline $\mathrm{D} \times 7$ & $0.00 \pm 0.00$ & $0.00 \pm 0.00$ & $0.00 \pm 0.00$ & $0.00 \pm 0.00^{\mathrm{a}}$ \\
\hline $\mathrm{E} \times 7$ & $0.00 \pm 0.00$ & $0.00 \pm 0.00$ & $0.00 \pm 0.00$ & $0.00 \pm 0.00^{\mathrm{a}}$ \\
\hline $\mathrm{A} \times 15$ & No construct & $0.00 \pm 0.00$ & $0.00 \pm 0.00$ & $0.00 \pm 0.00^{\mathrm{a}}$ \\
\hline $\mathrm{B} \times 15$ & $0.00 \pm 0.00$ & $0.00 \pm 0.00$ & $0.00 \pm 0.00$ & $0.00 \pm 0.00^{\mathrm{a}}$ \\
\hline $\mathrm{C} \times 15$ & $0.00 \pm 0.00$ & $0.50 \pm 0.55$ & $0.33 \pm 0.52$ & $0.83 \pm 0.02^{\mathrm{c}}$ \\
\hline $\mathrm{D} \times 15$ & $1.67 \pm 0.52$ & $1.67 \pm 0.52$ & $0.50 \pm 0.55$ & $3.84 \pm 0.02^{\mathrm{d}}$ \\
\hline$E \times 15$ & $0.00 \pm 0.00$ & $0.67 \pm 0.52$ & $0.17 \pm 0.41$ & $0.84 \pm 0.02^{\mathrm{c}}$ \\
\hline $\mathrm{A} \times 30$ & No construct & $1.17 \pm 0.41$ & $0.00 \pm 0.00$ & $1.17 \pm 0.41^{\mathrm{a}}$ \\
\hline $\mathrm{B} \times 30$ & $0.65 \pm 0.55$ & $0.85 \pm 0.52$ & $0.00 \pm 0.00$ & $1.50 \pm 0.02^{\mathrm{a}}$ \\
\hline $\mathrm{C} \times 30$ & $1.00 \pm 0.63$ & $1.50 \pm 0.55$ & $0.67 \pm 1.03$ & $3.17 \pm 0.26^{\mathrm{c}}$ \\
\hline $\mathrm{D} \times 30$ & $2.50 \pm 0.55$ & $3.17 \pm 1.33$ & $1.50 \pm 0.55$ & $7.17 \pm 0.45^{\mathrm{d}}$ \\
\hline$E \times 30$ & $1.50 \pm 0.55$ & $1.50 \pm 0.55$ & $1.67 \pm 1.37$ & $4.67 \pm 0.47^{\mathrm{c}}$ \\
\hline$A \times 60$ & No construct & $1.67 \pm 0.52$ & $0.00 \pm 0.00$ & $1.67 \pm 0.52^{\mathrm{a}}$ \\
\hline $\mathrm{B} \times 60$ & $1.50 \pm 0.55$ & $1.33 \pm 0.52$ & $0.55 \pm 0.55$ & $3.38 \pm 0.02^{\mathrm{a}}$ \\
\hline $\mathrm{C} \times 60$ & $1.67 \pm 0.52$ & $2.67 \pm 0.52$ & $2.33 \pm 0.82$ & $6.67 \pm 0.17^{\mathrm{c}}$ \\
\hline$D \times 60$ & $2.67 \pm 0.52$ & $3.50 \pm 0.84$ & $3.33 \pm 1.03$ & $9.50 \pm 0.26^{\mathrm{d}}$ \\
\hline$E \times 60$ & $2.67 \pm 0.52$ & $3.00 \pm 0.89$ & $2.00 \pm 1.26$ & $7.67 \pm 0.17^{\mathrm{e}}$ \\
\hline $\mathrm{A} \times 90$ & No construct & $2.00 \pm 0.00$ & $0.67 \pm 0.82$ & $2.67 \pm 0.82^{\mathrm{a}}$ \\
\hline $\mathrm{B} \times 90$ & $1.83 \pm 0.41$ & $2.33 \pm 0.52$ & $1.67 \pm 0.52$ & $5.83 \pm 0.06^{\mathrm{b}}$ \\
\hline $\mathrm{C} \times 90$ & $2.00 \pm 0.63$ & $3.50 \pm 0.55$ & $3.33 \pm 0.82$ & $8.83 \pm 0.14^{\mathrm{c}}$ \\
\hline $\mathrm{D} \times 90$ & $3.50 \pm 0.84$ & $3.83 \pm 0.41$ & $3.33 \pm 0.03$ & $10.66 \pm 0.23^{\mathrm{d}}$ \\
\hline $\mathrm{E} \times 90$ & $2.83 \pm 0.00$ & $3.17 \pm 0.75$ & $3.83 \pm 0.41$ & $9.83 \pm 0.03^{\mathrm{e}}$ \\
\hline
\end{tabular}



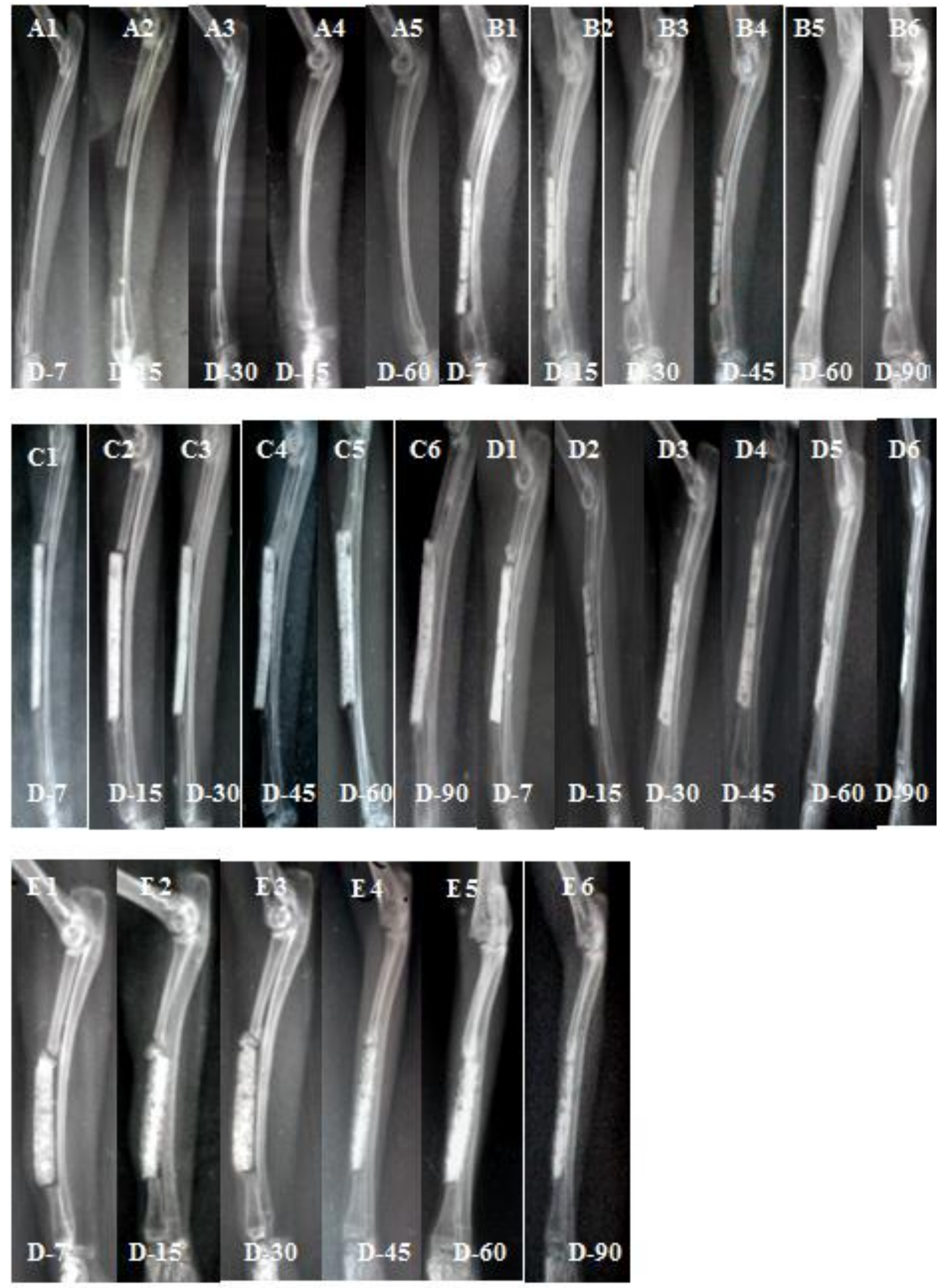

Figure 1: Mediolateral radiographs of groups $A, B, C, D$ and E at different intervals. A1: $\operatorname{gr} A$ at day 7, A2: $\operatorname{gr} A$ at day 15, A3: $\operatorname{gr} A$ at day 30, A4: $g r A$ at day 45, A5: $\operatorname{gr} A$ at day 60; B1: $\operatorname{gr} B$ at day 7, B2: $\operatorname{gr} B$ at day 15, B3: $\operatorname{gr} B$ at day 30, B4: $\operatorname{gr} B$ at day 45, B5: gr B at day 60, B6: $\operatorname{gr} B$ at day 90; C1: gr C at day 7, C2: gr Cat day 15, C3: $\operatorname{gr} C$ at day 30, C4: $\operatorname{gr} C$ at day 45, C5: $\operatorname{gr} C$ at day 60, C6: $\operatorname{gr} C$ at day 90; D1: $\operatorname{gr} D$ at day 7, D2: $\operatorname{gr} D$ at day 15, D3: $\operatorname{gr} D$ at day 30, D4: $\mathrm{gr} D$ at day 45,

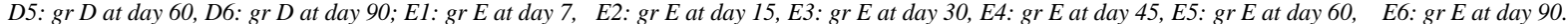




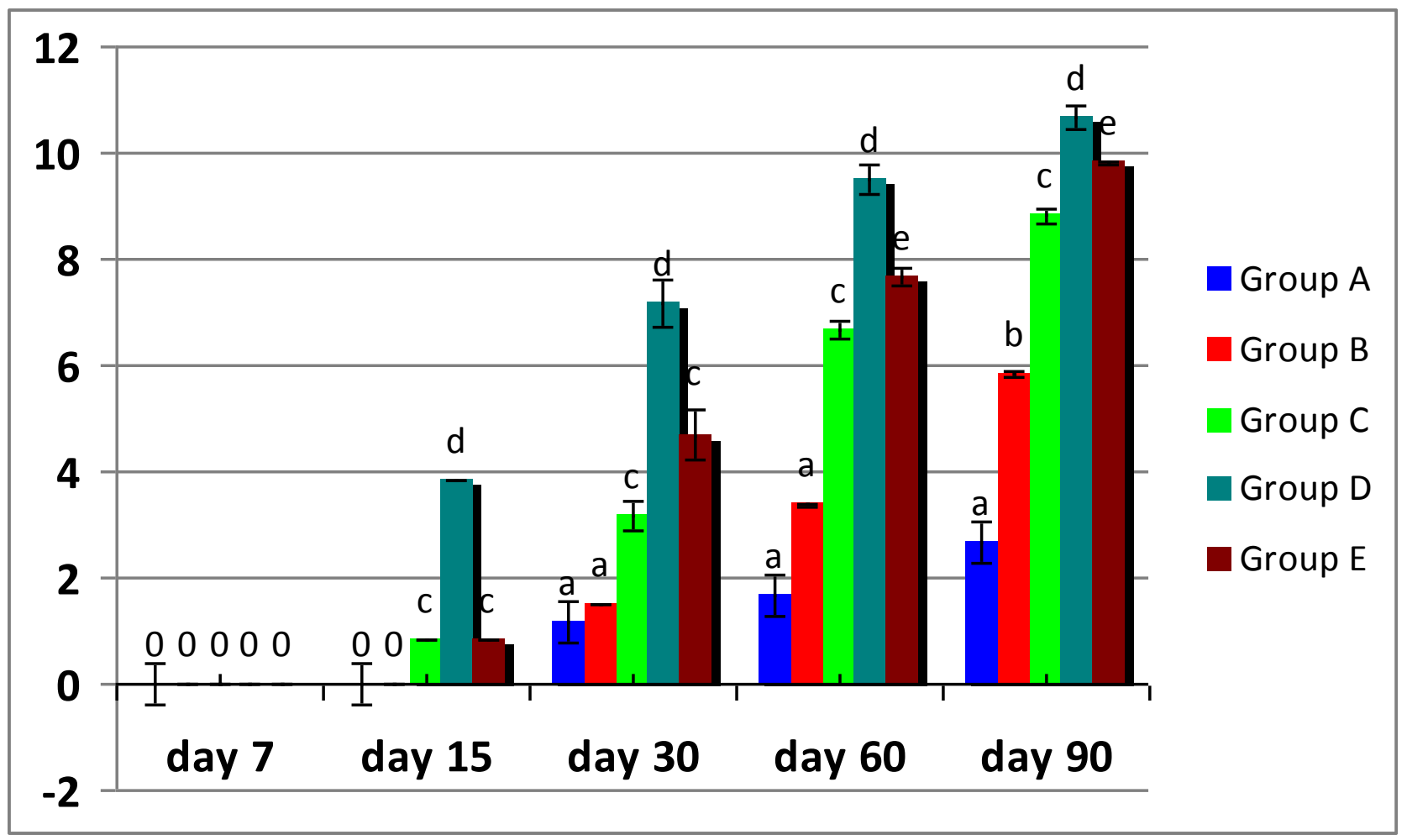

Figure 2: Histogram showing of total radiographic score $($ mean $+S D)$ in different groups at different time intervals

In control group (A), at day 30, the osteotomy line at defect site became invisible marking the progress of bone repair near this cut end. At day 45, a slight increase in radio-opacity was seen near this defect, marking the beginning of callus formation. On day 60 , the area of radio-opacity was seen to be spread a little more and the defect remaining unbridged. No animal of control group survived at day 90 as there was complete fracture of remaining ulna caused complete lameness and unable to stand or walk. In group B, mild resorption of the construct started at day 45 and at day 60 , the resorption was moderate. There was gap between the construct and the cut ends of host bone till day 60, but there was increased bone density at the junction of construct with the ulna at day 90 . There was no evidence of bridging at either proximal or distal end of ceramic block with the host bone. Osteogenic process was very negligible. In group $\mathrm{C}$, bridging of the bone and construct at proximal end was seen from day 30 onwards. At day 60, there was close attachment of the construct with the adjacent ulna and bridging between composite construct and cut end of the host bone. At day 90, good amount of new bone formation was noticed and the construct also underwent moderate resorption by this time. In group $\mathrm{D}$, bridging between host bone and implant in both proximal and distal interfaces was evident at day 30 . At day 45, there was close union between composite scaffold and the adjacent ulna. At day 60, a major portion of the construct underwent resorption and the boundary between implant and normal bone disappeared, which indicated that the implant had been replaced by early new bone. This was more pronounced at day 90 (AP view). In group E, there was bony union between scaffold and ulna on day 45, whereas, bridging of both the proximal and distal defect ends with the host bone was seen at day 60. Moderate resorption of construct and bridging with adjacent ulna was more evident at day 90 .

Statistical analysis of total radiographic score at day 15 showed that group D was significantly $(\mathrm{P}<0.05)$ different from other groups. On day 60, there was significant difference $(\mathrm{P}<0.05)$ between group $\mathrm{C}$ and group $\mathrm{E}$. On day 90, all groups showed significant $(\mathrm{P}<0.05)$ variation from each other. Overall radiographic score showed that group D had the highest score, followed by groups E, C, B and A.

\section{Micro Computed Tomography assessment}

Micro computed tomography assessment was able to distinguish between the three phases present inside the defects: newly formed bone, remaining triphasic bioceramic block and non-mineralized tissues. The $\mu \mathrm{CT}$ scanned slices of bone biopsy from different groups are presented in Figure 3. The transverse and longitudinal sections revealed the unbridged cut ends of the host radius and there was no osteogenic reaction in-between radius and adjacent ulna in any of the animal group A. In other groups, newly formed trabecular bone at the defect site in radius bone was seen. The HASi component in defect site was almost intact in group B, whereas it was in the process of resorption in groups $\mathrm{C}, \mathrm{D}$ and $\mathrm{E}$. In group $\mathrm{C}$, signs of osteogenesis and process of remodeling were observed. In group D, transverse and longitudinal bone sections showed the establishment of periosteal continuity in between the cut ends of old host bone (radius) and continuation of medullary cavity at the defect site. Newly formed trabecular bone was under remodeling process in groups $\mathrm{D}$ and $\mathrm{E}$.

\section{Histopathological assessment}

Histopathological sections at bone defect site of different groups at day 90 are presented in Figure 4. In control group (A) majority of the defect area was occupied by fibrous tissue (Figure 4A). In group $B$, very little new woven bone formation, which was discontinuous in nature, was seen at the junction of scaffold and cut ends of the host bone (Figure 4B). The scaffold was in the process of resorption and localized regions of new bone formation were seen as dispersed islands. In group $\mathrm{C}$, new woven bone formation at the junction of scaffold and cut ends of host bone and osteochondral union between the ulna and scaffold was seen (Figure 4C). There was invasion of periosteum from the adjacent ulna into the defect area. 

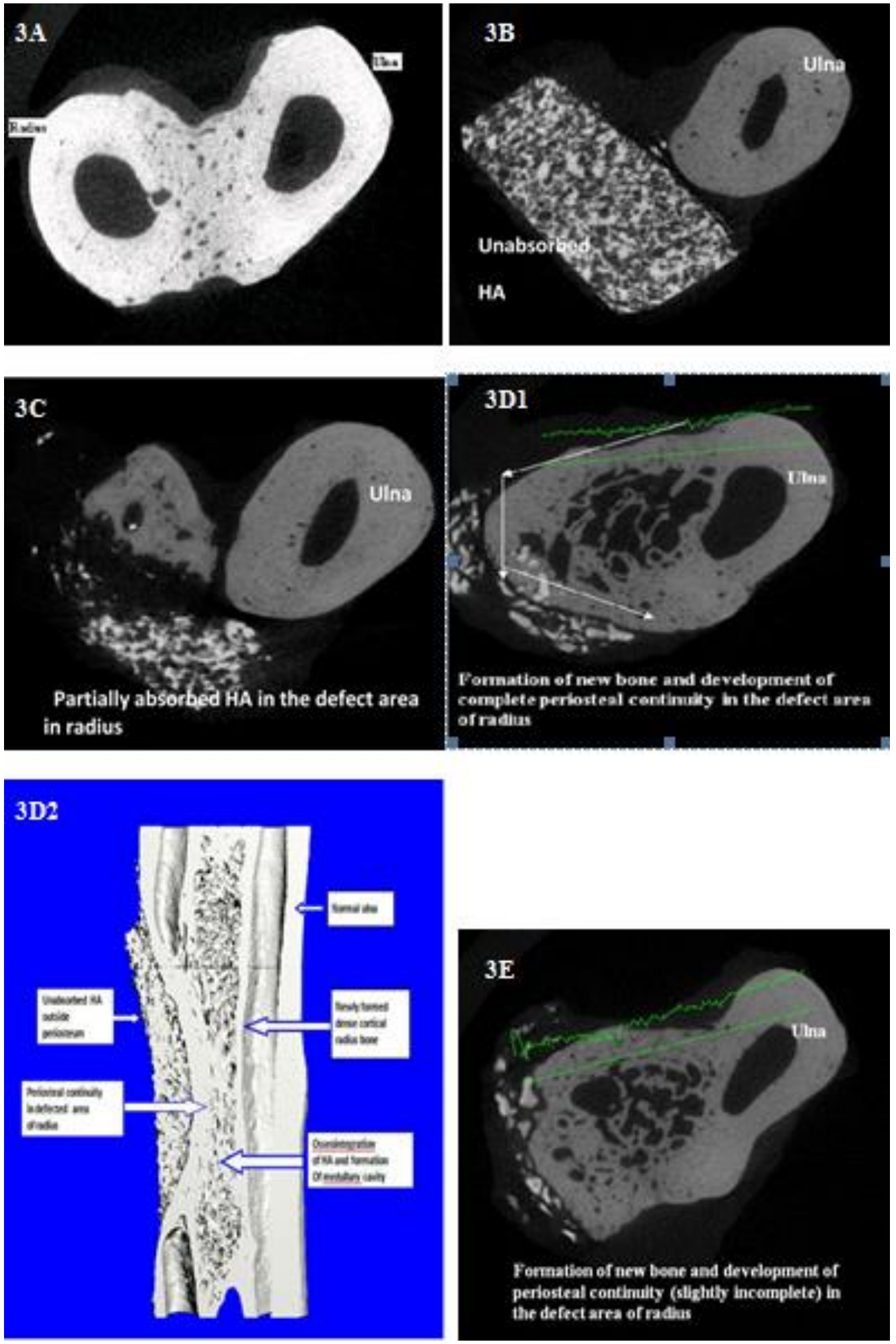

Figure 3: 3D transverse bone biopsy slice of groups $A, B, C, D$ and $E$ at day 90. 3A: normal bone biopsy of gr $A, 3 B$ : bone biopsy slice of gr $B, 3 C$ : bone biopsy slice of $\mathrm{gr} C$, 3D1\& 3D2: bone biopsy slice of gr $D$, 3E bone biopsy slice of $\mathrm{gr} E$ 

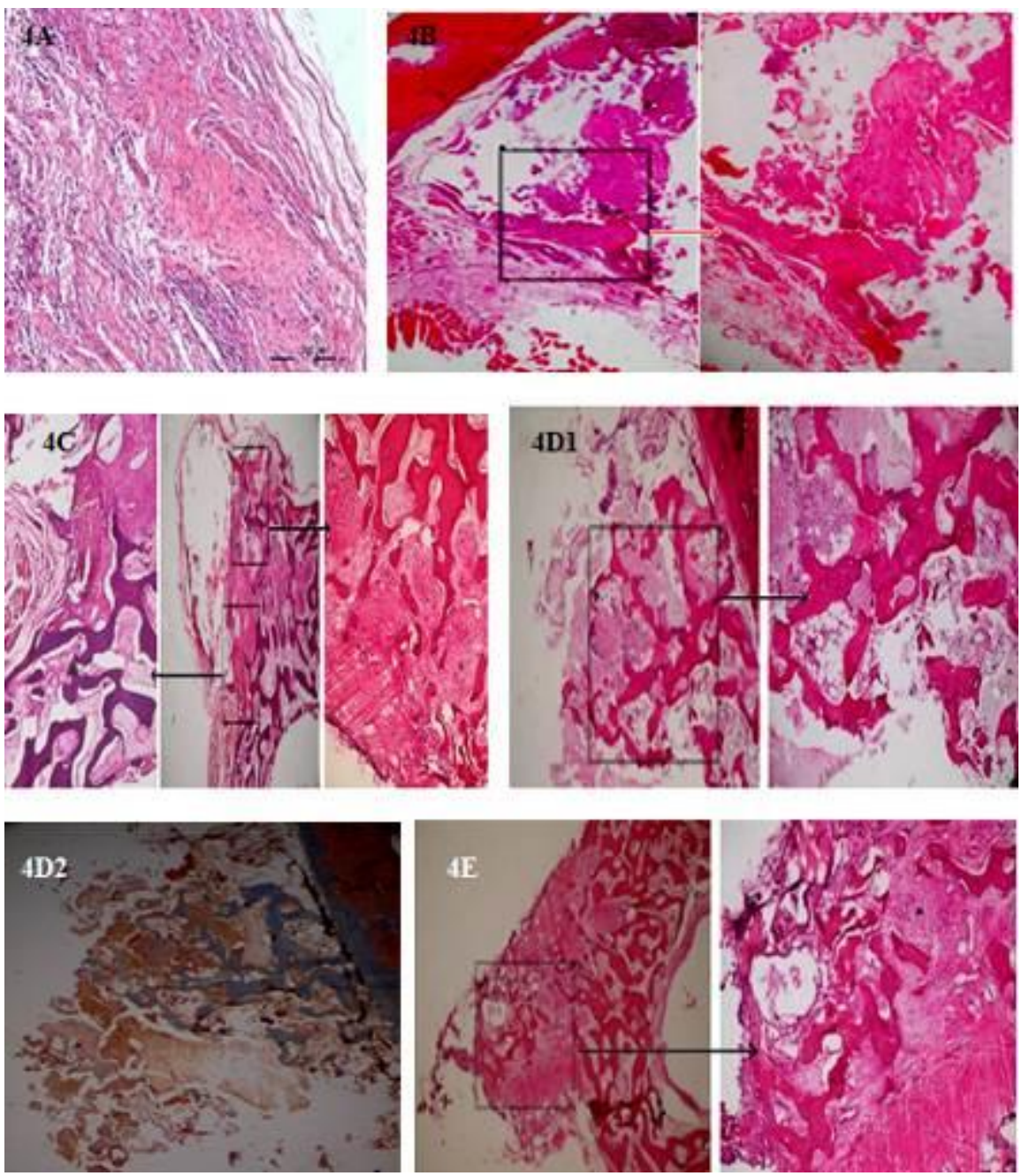

Figure 4: Histopathological section at bone defect site of different groups at day 90; 4A: Longitudinal section gr A showed fibrous tissue proliferation at the cut ends, H\&E, 100X; 4B: Transverse section of gr B showed few areas of new bone formation at the junction of scaffold and host bone, H\&E, 40X, 100X; 4C: Transverse section of gr $C$ showed osteochondral union between the ulna and scaffold, H\&E, 40X, 100X; 4D1: Transverse section of gr D showed extensively bony union of scaffold with adjacent ulna, H\&E, 40X, 100X; 4D2: Transverse section of gr D showed newly formed bone in the defect area repreasented by areas of mineralization (blue staining) and new osteoid (deep red staining) Masson's Trichome, 40x; 4E: Transverse section of gr E showed osteochondral union between the ulna and scaffold, H\&E, 40X, 100X.

Table 4. The total histological scores (mean \pm SD) of bone healing in different groups. a-e values with different superscript letters vary significantly in the last column.

\begin{tabular}{|c|c|c|c|c|c|c|}
\hline Group & Osteogenesis & Union & Marrow & $\begin{array}{c}\text { Cancellous/medullary } \\
\text { bone }\end{array}$ & $\begin{array}{c}\text { Cortical/compact } \\
\text { bone }\end{array}$ & Total score \\
\hline A & $1.00 \pm 0.00$ & $1.33 \pm 0.00$ & $0.00 \pm 0.00$ & $0.00 \pm 0.00$ & $0.00 \pm 0.00$ & $2.33 \pm 0^{\mathrm{a}}$ \\
\hline B & $2.00 \pm 0.00$ & $1.33 \pm 1.15$ & $0.67 \pm 0.58$ & $0.67 \pm 0.58$ & $0.00 \pm 0.00$ & $4.67 \pm 0.33^{\mathrm{b}}$ \\
\hline C & $1.67 \pm 0.58$ & $2.66 \pm 0.58$ & $0.33 \pm 0.58$ & $1.33 \pm 0.58$ & $0.00 \pm 0.00$ & $5.99 \pm 0.21^{\mathrm{c}}$ \\
\hline D & $3.00 \pm 0.00$ & $3.00 \pm 0.00$ & $2.00 \pm 0.00$ & $3.00 \pm 0.00$ & $1.66 \pm 0.58$ & $12.66 \pm 0.58^{\mathrm{d}}$ \\
\hline E & $2.33 \pm 0.58$ & $2.33 \pm 0.58$ & $1.00 \pm 0.00$ & $2.00 \pm 0.00$ & $0.00 \pm 0.00$ & $7.66 \pm 0.33^{\mathrm{e}}$ \\
\hline
\end{tabular}


The scaffold was under active resorption and replaced by newly formed woven bone at some places. In group D, abundant new dense bone tissue formation and close bony union of scaffold with the host bone at both cut ends as well as with the adjacent ulna was seen (Figure 4D1). Scaffold was under active resorption and many places it was replaced by newly formed woven bone. Increased vascularisation near the junction between scaffold and the adjacent ulna was seen. Marrow formation was also evident in more than half of the implant area indicative of remodeling. Osteogenesis process was of intramembraneous type without any intermediate cartilage formation. Masson's trichrome staining revealed close association of newly formed bone in the defect area with the host bone represented by more areas of mineralization (blue staining) and new osteoid (deep red staining) tissue (Figure 4D2). In group E, osteochondral union was seen between the scaffold and cut ends of host bone as well as with the ulna (Figure 4E). Three-fourth of the scaffold had undergone cartilaginous transition and areas of new bone formation were evident. The total histological scores of bone healing for different groups are presented in Table 4. Group D had the highest score, followed by groups E, C, B and A.

\section{Discussion}

The aim of the present work was to study the effect of BMSC with growth factors (rhBMP-2 and IGF-1) seeded on a resorbable bioceramic scaffold (HASi) in the regeneration of bone at very large critical-sized defect, using an autogenous approach.

The rabbit radial critical-sized defect creates a weight bearing model in which the implanted scaffold will sensitize the surrounding mechanical load and act as a mechanical and structural rapport to the defect. In addition, since the ulna can also serve as an internal splint for the radius, no external fixation is needed. Even then, in this study since the defect size was too large, we used a custom-designed external splint which was maintained for 7 days. Transplantation of mesenchymal stem cells for bone regeneration in a rabbit critical-size defect $(15 \mathrm{~mm})$ has been already reported ${ }^{[5]}$, but the regeneration potential of autogenous MSC with or without growth factors beyond critical-size defect was not reported so far. So, in this study, we created large $(30 \mathrm{~mm})$ segmental radial diaphyseal bone defect in rabbit model and we found that this model was very much suitable for this experiment. The only limitation of this model was without any scaffold or external fixation animal was unable to walk properly.

No significant bone formation and consolidation of the defect was achieved in any animal in which the defect was left untreated (control group). Since the purpose of the present study was to investigate the influence of MSC with or without growth proteins; empty scaffolds were used as an additional control group. The scaffold used in the present study was HASi-a triphasic bio-ceramic adding silicon in hydroxyapatite and tricalcium phosphate. In the bio-ceramic group (B) there was no evidence of bridging at either proximal or distal end of ceramic block with the host bone at day 90 post implantation. Osteogenic process was very negligible. Silica coated hydroxyapatite has osteoinductive properties ${ }^{[21]}$, in addition to faster resorption rate resulting from the greater dissolution of $\mathrm{Si}$ ions ${ }^{[10]}$. Nevertheless, with regard to the results of the present study, HASi alone is not able to lead to healing of large critical-size-defects. Although, more new bone formation could be seen in animal treated with HASi alone compared with the empty controls, but it failed to bridge the defect even at day 90 post implantation. Similarly, reconstruction of $1.5 \mathrm{~cm}$ segmental bone defects in the rabbit ulna using porous polylactic-coglycolic acid (PLGA) scaffold failed to bridge completely even at 12 weeks post implantation ${ }^{[22]}$. In our view, even if this bio-ceramic implant (HASi) or other bio-implants include hydroxyapatite (HA) or its combination of tri-calcium phosphate (HA-TCP) or PLGA kept for further longer duration, it might be failed to bridge the large bone defect completely. For this, further study wad suggested using different bio-materials for implantation in very large $(3 \mathrm{~cm})$ radial bone defect in rabbit model.

In an additional control group, a combination of autogenous MSC and HASi was transplanted into the defect area. In these animals, increased bone formation was found compared with HASi alone. Radiological, $\mu \mathrm{CT}$ and histological analysis showed bony union between the scaffold and cut ends of the host bone as well as with the adjacent ulna. This observation confirms previous in vivo and in vitro experiments which demonstrated the benefit of the addition of mesenchymal stem cells on bio-scaffold for improved bone regeneration ${ }^{[3,5]}$. Mesenchymal stem cells (MSCs) are nonhematopoietic stromal stem cells, capable of self-replication and of differentiating into, and contributing to the regeneration of mesenchymal tissues such as bone, cartilage, ligament, tendon, muscle and adipose tissue. MSCs promote infiltration of osteoprogenitor cells, and thus enhance their subsequent mineralization and bone formation ${ }^{[22,23]}$. MSC-derived osteoblast cells are anchorage dependent and require a supportive matrix in order to survive and flourish. So the selection of a suitable scaffold is another important criterion to be considered to fabricate a tissueengineered construct. Silica coated hydroxyapatite scaffold (HASi) has significantly increased cell viability, proliferations and differentiation of osteoblast from different source in an in vitro culture $^{[9]}$. The enhanced viability and proliferation of cells on HASi can be attributed to the multiphase composition (HA, tricalcium phosphate and calcium silicate) as well as silica content of $\mathrm{HASi}^{[21]}$. Silica-coated hydroxyapatite (HASi) could induce the osteogenic differentiation of rabbit BMSC by day 17 post-seeding in absence of any osteogenic induction media ${ }^{[24]}$. Since HASi has been provided a favorable $3 \mathrm{D}$ microenvironment for the osteogenic response, our aim was to use this tissue-engineered construct for bone regeneration and repair in a very large radial segmental defects in rabbits. Generally, cell proliferation and differentiation are two interdependent processes having a counteracting relationship. However, HASi was capable of inducing osteogenesis and cell proliferation in a parallel relationship, when it seeded with human BMSC ${ }^{[9]}$. This is in confirmation of our study where rabbit BMSC-seeded HASi scaffold promotes proliferation and osteogenic differentiation of mesenchymal stem cells in segmental bone defects.

In vivo bone formation can be influenced by the type and form of biomaterial, the pore size and pore distribution, interconnectivity and the resorption of the bi-omaterial ${ }^{[25]}$. The bio-material used (HASi) in our study was manufactured in blocks, consequently this cannot be compared to other studies where granules or powders were used ${ }^{[5]}$. For example Krebsbach et $a l^{[26]}$ found more bone formation with HA/TCP powder than in HA/TCP blocks. In our study, blocks were used as scaffolds because of the better bio-mechanics and the easier future clinical use in segmental bone defects ${ }^{[3]}$. The bio-material used in the present study comprised open pores in the range of 50-500 $\mu \mathrm{m}$, and geometry wise; it is fully interconnected with a large surface area to volume ratio. The latter quality to have seems to have improved the migration and distribution of osteoprogenitor cells throughout the scaffold material. Secretion of osteoclasts is stimulated by Si ions and this in turn confirms the osteointegrative, osteoconductive and degradation properties of triphasic HASi bio-ceramic scaffold ${ }^{[27]}$. In this study, HASi block seems inevitability causing close union between scaffold and adjacent ulna due to its osteoconductive properties, which is seems to be good as it not only unite the adjacent splint bone near the defect site, also it provided further mechanical stability to the injured bone.

Bone development, growth, and repair predominantly occur through 
the process of endrochondral ossification, characterized by remodeling of cartilaginous templates. The same route efficiently supports engineering of bone marrow as a niche for hematopoietic stem cells-mesenchymal stem cells (MSC) ${ }^{[28]}$. Mesenchymal stem cells are known to have the ability to undergo osteogenic differentiation under suitable conditions ${ }^{[29]}$. Their proliferation and differentiation capacity may be enhanced by exposing them to growth factors. Growth factors are protein signaling agents that act locality to stimulate formation and proliferation of osteoblasts and thereby promote bone healing. The rhBMP-2-treated constructs in group D encouraged the formation of abundant bone tissue bridging both proximal and distal interfaces as well as with adjacent ulna as early as eight weeks after surgery. At day 90 after surgery, $\mu \mathrm{CT}$ and histopathological analysis showed newly formed bony tissue within the implantation site, well-differentiated mineralized lamellar bone surrounding the HASi granules, characterized by the number of osteocytic lacunae and Haversian systems and the presence of osteoid border at the surface of new bony trabeculae. Periosteal continuity was complete at the defect site with host bone. Direct ossification of the scaffold and dense woven bone along with marrow formation were also noticed in some animals of this group, suggest the possibility of direct formation of osteoblasts from the osteoprogenitor cells by the action of rhBMP-2. The results confirmed that rhBMP-2 accelerate osteogenesis at the defect site by the process of osteoinduction.

BMP-2 has the highest osteoinductive potential among all the bone morphogenetic proteins ${ }^{[1]}$. There are differences between species in their sensitivity to bone morphogenetic proteins, so the result can be difficult to extrapolate from one species to another ${ }^{[30]}$. A minimum threshold dose of BMP-2 is necessary for a beneficial effect to occur, but a higher dose does not necessarily result in a better outcome ${ }^{[13,31]}$. Bone morphogenetic proteins (BMPs) are hydrophobic, non-species specific glycoprotein, belongs to the expanding transforming growth factor-beta super family and has pleiotropic function that range from extra skeletal and skeletal organogenesis to bone generation and regeneration. It induces de novo bone formation in post fetal life through the process of direct (intra membranous) and endrochondral ossification and their response is dose dependent ${ }^{[32]}$. It is reported that a 2-cm ostectomy corresponds to a critical-size defect that does not heal spontaneously without the addition of bone grafts or any bone substitute $^{[1]}$. Such an experimental non-union model provides very limited osteoconduction as bone contact was only present at both proximal and distal cortical junctions. The rhBMP-2 appeared to have osteoinductive properties that were quite efficient in such surgical conditions, and combining with this material (HASi) might ensure early healing. Bone morphogenetic protein- 2 accelerates the rate of development of the callus and cortical union after fracture of the rabbit tibia, but it does not affect the amounts of bone and cartilage produced. Bone morphogenetic protein-2 stimulates bone and cartilage formation so that the callus develops and matures more rapidly ${ }^{[33]}$. In agreement with this study, recombinant human bone morphogenetic protein-2 accelerates healing in a rabbit ulnar osteotomy model $^{[34]}$.

It appears difficult to decide on an ideal concentration, since these studies varied in term of the BMP-2 carrier and the size of the defect. In this study, the same number of rBMSC (five millions cells) and concentration of growth factors rhBMP-2 $(50 \mu \mathrm{g})$ and IGF-1 $(50 \mu \mathrm{g})$ were again injected around the bone defect on day 15 postsurgery in groups $\mathrm{C}, \mathrm{D}$ and $\mathrm{E}$ respectively, as the defect size was almost double than standard radial critical-size bone defect $(15 \mathrm{~mm})$ in rabbit model $^{[5]}$. In our study, local injection of $100 \mu \mathrm{g}$ rhBMP-2 allowed bone regeneration in an extensively large $(30 \mathrm{~mm})$ radial diaphyseal critical-size defect in rabbit model. It is reported that rhBMP-2 when injected $(120 \mu \mathrm{g})$ at the site of bone defect accelerates the rate of development of callus and cortical union after fracture of the rabbit tibia and ulna ${ }^{[33,34]}$. However, further experiment was needed for examining the beneficial effect of rhBMP-2 injected locally. Further studies also needed to assess the kinetic release of rhBMP-2 from our HASi construct.

Histological and $\mu \mathrm{CT}$ analysis in group $\mathrm{E}$ revealed that there was osteochondral union and cartilaginous transition between implant and host bone at defect site as well as with the adjacent ulna. Completion of cancellous bone formation and regions of increased neovascularisation with moderate resorption of construct took place at day 90 post surgery. New bone formation was more than group C, but comparatively less than group D. This could be because of IGF-1 is known to induce stem cells to differentiate towards the endothelial precursor germ layer, with significantly more endothelial cells at later stages ${ }^{[35]}$. There was cartilaginous transition of scaffold and new bone formation in group E as IGF-1 increases collagen and noncollagenous protein synthesis in vitro ${ }^{[36,37,38]}$. Previous studies also showed the ability of IGF to promote in vivo bone healing ${ }^{[39,40]}$. Insulin-like growth factor-1 plus MSCs can improve bone healing mostly through endrochondral ossification ${ }^{[41]}$.

Among the five groups, in vivo study showed that a radial segmental defect of $30 \mathrm{~mm}$ did not show any sign of osteogenesis or bone healing in control (left untreated) group. There was little sign of osteogenesis in scaffold group; however, it provided very good mechanical as well as structural support to the defect, but it also unable to bridge the bone defect at day 90 post implantation. In scaffold-MSC seeded group, osteogenesis was better than the group where only scaffold was applied, probably due to the addition of MSCs, which promote infiltration of osteoprogenitor cells, and thus enhance their subsequent mineralization and bone formation. Addition of growth factors in scaffold-MSC construct accelerated the healing of critical sized bone defect. Among the two growth proteins, rhBMP-2-treated constructs encouraged the formation of abundant bone tissue bridging both proximal and distal interfaces as well as with adjacent ulna as early as eight weeks after surgery. IGF-1 treated construct also encourage new bone formation and bridging the defect but osteogenesis was comparatively less than rh-BMP-2 treated group. The HASi was acted as suitable bioscaffold for development of MSC-seeded tissue engineering construct for bone regeneration because of their biocompatibility and osteoconductivity.

\section{Conclusion}

The findings of this study shows that in vitro seeding of autogenous rBMSC on bioscaffold HASi could hasten the osteoconductive properties of scaffold and can be alternative to autogenous bone graft in the treatment of large bone defects and non-union. Addition of growth proteins particularly rhBMP-2 to autogenous MSC seeded HASi bio-ceramic construct could accelerate bone regeneration in a large critical-size-bone defect model controlled study.

\section{References}

1. Minier K, Toure A, Fusellier M, Fellah B, Bouvy B, Weiss P, Gauthier O. BMP-2 delivered from a self-cross linkable $\mathrm{Ca} / \mathrm{P}$ hydrogel construct promotes bone regeneration in a critical-size segmental defect model of non-union in dogs. Vet Comp Orthop Traumatol 2014; 27 (6): 411-21.

2. Li Ye, Chen SK, Li L, Qin L, Wang XL, Lai YX. Bone defect animal models for testing efficacy of bone substitute biomaterials. J Orthop Translatiom 2015; 3(3): 95-104

3. Kasten P, Vogel J, Luginbuhl R, Niemeyer P, Tonak M, Lorenz H. Ectopic bone formation associated with mesenchymal stem cells in a resorbable calcium deficient hydroxyapatite carrier. Biomaterials 2005; 26(29): 5879-89.

4. Bruder SP, Kraus KH, Goldberg VM, Kadiyala S. The effect of implants loaded with autologous mesenchymal stem cells on the healing of canine segmental bone defects. J Bone Joint Surg Am 1998; 80(7):985-96. 
5. Niemeyer P, Szalay K, Luginbuhl R, Sudkamp NP, Kasten $P$ Transplantation of human mesenchymal stem cells in a nonautogenous setting for bone regeneration in a rabbit critical -sizedefect model. Acta Biomaterialia 2010; 6 (3): 900-08.

6. Kim HJ, Park JB, Lee JK, Park EY, Park EA, Riew KD, Rhee SK. Transplanted xenoxenic bone marrow stem cells survive and generate new bone formation in the poterolateral lumber spine of non-immunosuppressed rabbits. Eur Spine 2008; 17 (11): 1515-21.

7. Daculsi G, Passuti N, Martin S, Deudon C, Legeros RZ, Raher S Macroporous calcium phosphate ceramic for long bone surgery in humans and dogs. Clinical and histological study. J Biomed Mater Res 1990; 24 (3):379-96.

8. Baur TW, Smith ST. Bioactive materials in orthopedics surgery: overview and regulatory considerations. Clin Orthop Rev 2002; 395(2):11-22

9. Nair MB, Bernhardt A, Lode A, Heinemann C, Thieme S, Hanke T, Varma H, Gelinsky M, John A. A bioactive triphasic ceramiccoated hydroxyapatite promotes proliferation and osteogenic differentiation of human stromal cells. J Biomed Mater Res A 2009a; 90 (2): 533-42.

10. El-Ghannam AR. Advanced bioceramic composite for bone tissue engineering: design principles and structure-bioactivity relationship. J Biomed Mater Res A 2004: 69 (3): 490-501.

11. Linkhart TA, Mohan S, Baylink DJ. Growth factors for bone growth and repair: IGF, TGF-beta and BMP. Bone 1996; 19 (1 Suppl): 1S-12S.

12. Devescovi V, Leonardi E, Ciapetti G, Cenni E. Growth factors in bone repair. Chir Organi Mov 2008; 92(3):161-68

13. Sciadini MF, Jojnson KD. Evaluation of recombinant human bone morphogenetic protein-2 as a bone-graft substitute in a canine segmental defect model. J Orthop Res 2000; 18 (2):289-302.

14. Pluhar GE, Manley PA, Heiner JP, Vanderby R Jr, Seeherman HJ, Markel MD. The effect of recombinant human bone morphogenetic protein -2 on femoral reconstruction with an intercalary allograft in a dog model. J Orthop Res 2001; 19 (2): 308-17

15. Fiedler J, Brill C, Blum WF, Brenner RE. IGF-1 and IGF-2 stimulate directed cell migration of bone marrow derived human mesenchymal progenitor cells. Biochem Biophys Res Comm 2006; 345 (3): 1177-83

16. LeGeros RZ. Properties of osteoconductive biomaterials: calcium phosphates. Clin Orthop Relat Res 2002; 395 (2): 81-98.

17. Maiti SK, Shiva Kumar MU, Srivastava L, Kumar N, Ninu AR. Isolation, proliferation and morphological characteristics of bone marrow derived mesenchymal stem cells (BM-MSC) from different animal species. Trends Biomater Artif Org 2012; 27(1): 29-35.

18. Yang, C.Y., Simmons. D.J. and Lozano. R. The healing of grafts combining freeze-dried and demineralized allogeneic bone in rabbits. Clin Orthop Relat Res 1994; 298 (1):286-95.

19. Lane, J. M. and Sandhu, H. S. Current approach to experimental bone grafting. Orthop Clin North Am 1987; 18(2): 213-25.

20. Heiple, K. G., Goldberg, V. M., Powell, A. E., Bos, G. D. and Zika, J. M. Biology of cancellous bone grafts. Orthop Clin North Am 1987: 18(2): 179-85.

21. Nair MB, Varma HK, Menon KV, Shenoy SJ, John A. Reconstruction of goat femur segmental defects using triphasic ceramic-coated hydroxyapatite in combination with autologous cells and platelet-rich plasma. Acta Biomater 2009c; 5 (5): 174255 .

22. Zhang X, Qi YY, Zhao TF, Li D, Dai X S, Niu L, He RX Reconstruction of segmental bone defects in the rabbit ulna using periosteum encapsulated mesenchymal stem cells-loaded poly (lactic-co-glycolic acid) scaffolds. Chin Med J 2012; 125 (22): 4031-36.

23. Wang X, Wang Yu, Gou W, Lu Q, Peng J, Lu S. Role of mesenchymal stem cells in bone regeneration and fracture repair: a review. Int Orthop 2013; 37 (12): 2491-98.
24. Ninu AR, Maiti SK, Palakkara S, Kritaniya D, Mahan T, Kumar N In vitro osteoinduction potential of a novel silica coated hydroxyapatite bioscaffold seeded with rabbit mesenchymal stem cell. J Stem Cell Res Ther 2016; 2(1): 00009.

25. Mankani MH, Kuznetsov SA, Fowler B, Kingman A, Robey PG. In vivo bone formation by human bone marrow stromal cells: effect of carrier particle size and shape. Biotechnol Bioeng 2001; 72 (1): 96107.

26. Krebsbach PH, Kuznetsov SA, Satomura K, Emmons RV, Rowe DW, Robey PG. Bone formation in vivo: comparison of osteogenesis by transplanted mouse and human marrow stromal fibroblasts. Transplantation 1997; 63 (8):1059-69..

27. Nair MB, Varma HK, Menon KV, Shenoy SJ, John A. Tissue regeneration and repair of goat segmental femur defect with bioactive triphasic ceramic-coated hydroxyapatite scaffold. J Biomed Mater Res A 2009b; 91(3): 855-65.

28. Scotti C, Tonnarelli B, Papadimitropoulos A, Piccinini E, Todorov A, Centola M, Barbero A, Martin I. Engineering small-scale and scaffold-based bone organs via endochondral ossification using adult progenitor cells. Methods Mol Biol 2016; 1416: 413-14.

29. Birmingham E, Niebur GL, McHugh PE, Shaw G, Barry FP, McNamara LM. Osteogenic differentiation of mesenchymal stem cells in regulated by osteocyte and osteoblast cells in a simplified bone niche. Cell Mater 2012; 23 (1):13-27

30. Itoh T, Mochizuki M, Nishimura R, Matsunaga S, Kadsawa T, Kobuko S, Yokota S, Sasaki N. Repair of ulnar segmental defect by recombinant human bone morphogenetic protein -2 in dogs. J Vet Med Sci 1998; 60:451-58.

31. Boraish S, Paul O, Hawkes D, Wickham M, Lorich DG Complications of recombinant human BMP-2 for treating complex tibial plateau fractures: a preliminary report. Clin Orthop Relat Res 2009; 467 (12):3257- 62.

32. Maiti S K, Singh G R. Bone morphogenetic proteins-novel regulators of bone formation. Indian J Exp Biol 1998; 36 (3): 237-44

33. Bax BE, Wozney JM, Ashhurst DE. Bonemorphogenetic protein2increases the rate of callus formation after fracture of the rabbit tibia. Calcif Tissue Int 1999: 65 (1): 83-89.

34. Bouxsein ML, Turek TJ, Blake CA, D'Augusta D, Li X, Stevens M, Seeherman HJ, Wozney JM. Recombinant human bone morphogenetic protein-2 accelerates healing in a rabbit ulnar osteotomy model. J Bone Joint Surg Am 2001; 83(8): 1219-30.

35. Piecewicz SM, Pandey M, Roy B, Xiang SH, Zetter Br, Sengupta S. Insulin-like growth factors promote vasculogenesis in embryonic stem cells. PloS ONE 2012; 7 (2): e32191.

36. Canalis E, McCarthy T, Centrella M. Isolation and characterization of insulin like growth factor 1 (Somatomedin-C) from cultures of fetal rat calvariae. Endocrinology 1988; 122(1):22-27.

37. Hock JM, Centella M, Canalis E. Insulin-like growth factor-1 has independent effects on bone matrix formation and cell replication. Endocrinology 1988; 122 (1): 254-60.

38. McCarthy TL, Centella M, Canalis E. Insulin-like growth factor and bone. Connect Tissue Res 1989; 20(1): 277-82.

39. Schmidmaier G, Wildemann B, Bail H, Luke M, Fuchs T, Stemberger A, Flyvbjerg A, Hass NP, Raschke M. Local application of growth factors Insulin-like growth factor and transforming growth factor-beta-1 from a biodegradable poly (D, L-Lactide) coating of osteosynthetic implants accelerates fracture healing in rats. Bone 2001; 28(4):341-50

40. Blumenfeld I, Srouji S. Lanir Y, Laufer D, Livne E. Enhancement of bone healing in old rats by TGF-beta and IGF-1. Expt Geronto 2002; 37(4):553-65.

41. Myers TJ, Yan Y, Granero-Molto F, Weis JA, Longobardi L, Li T, Li Y, Contaldo C, Ozkan H, Spagnoli A. Systemically delivered insulin-like growth factor-1 enhances mesenchymal stem celldependant fracture healing. Growth Factors 2012; 30 (4):230-41. 


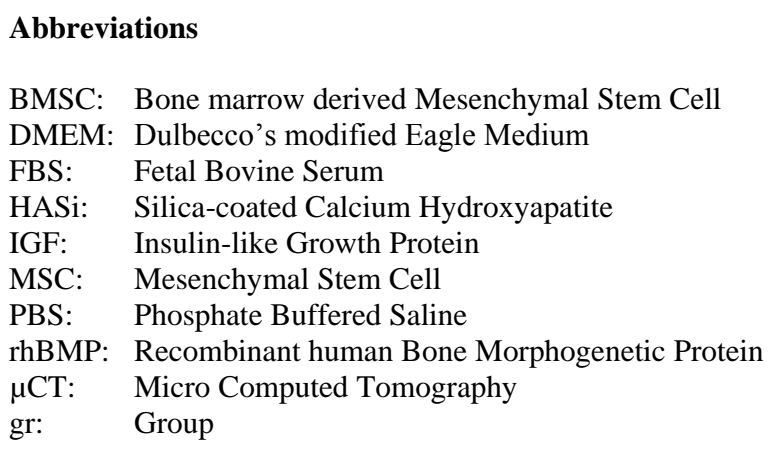

\section{Potential Conflicts of Interests}

\section{None}

\section{Acknowledgements}

The authors wish to thank Dr K. P. Singh, Principal Scientist, CADRAD, IVRI, Izatnagar (UP), India for his technical assistance in evaluation of histological sections and Prof. H. Varma, Sree Chitra Tirunal Institute for Medical Sciences and Technology, Trivandrum, Kerala (India) for his technical assistance for designing and preparation of HASi bio-ceramic. Senior author, wish to acknowledge to Prof Dimitry Spitkovsky, Institute of Neurophysiology, Köln University, Germany for his whole-hearted stem cell research support during his stay at Germany as "Visiting Professor".

This research was supported by a research grant from Department of Biotechnology (DBT), Government of India.

\section{Corresponding Author}

Swapan Kumar Maiti, Principal Scientist, Surgery Division, Indian Veterinary Research Institute, Izatnagar-243 122, Uttar-Pradesh, India Email:swapanivri@gmail.com; maiti_62@rediffmail.com 